\title{
THE VALUE OF SCREENING TESTS IN THE DETECTION OF PROSTATE CANCER. PART I: RESULTS OF A RETROSPECTIVE EVALUATION OF 1726 MEN* $^{*}$
}

\author{
CHRIS H. BANGMA, M.D., RIES KRANSE, ING., BERT G. BLIJENBERG, PH.D., \\ AND FRITZ H. SCHRÖDER, M.D., PH.D.
}

\begin{abstract}
Objectives. The ratio between free and total prostate-specific antigen (PSA) in serum (F/T ratio) was shown to improve the differentiation between prostate carcinoma and benign conditions in selected series of patients. In this study the F/T ratio was analyzed for its ability to improve the specificity of total serum PSA, digital rectal examination (DRE), and transrectal ultrasonography (TRUS) for the detection of prostate cancer in an unselected screening population of men identified in the Rotterdam population.

Methods. In 1726 men between 55 and 76 years old, 67 prostate carcinomas were detected by DRE, TRUS, and total serum PSA (Abbott IMx, Hybritech Tandem E). The DELFIA ProStatus PSA EOM and ProStatus PSA Free/Total assays (Wallac) were applied in retrospect to determine total and free serum PSA. Age, total prostate and inner zone volumes were taken into consideration.

Results. Sixty-seven carcinomas were detected, two by TRUS and three by DRE alone. Total serum PSA was the most important single predictor of prostate cancer, followed by DRE. The F/T ratio increased the specificity of total serum PSA in the PSA range between 4.0 and $10.0 \mathrm{ng} / \mathrm{mL}$. However, this improved specificity was not significant, nor for gland volumes restricted to $50 \mathrm{~mL}$ or less.

Conclusions. The combination of total serum PSA and DRE remains the standard for detection of prostate carcinoma in a screening population. Their specificity may be improved minimally by the F/T ratio, but not significantly in a sample of 1726 screened men. The threshold of the F/T ratio, and the optimal PSA range for its application, remains to be assessed prospectively. UROLOGY ${ }^{\circledR}$ 46: 773-778, 1995.
\end{abstract}

$S^{c}$ reening for prostate cancer (PC) has been performed from June 1994 onward in the Rotterdam area as part of the European Randomised Study of Screening for Prostate Carcinoma (ERSPC) to study the usefulness of early detection in the general population.

Selection of candidates for prostate biopsy is complicated by the fact that all screening modalities lack sufficient specificity. This results in a high ratio between the number of biopsies and the number of detected carcinomas. In most screening studies, overall about five biopsies are needed to detect one carcinoma. ${ }^{1-5}$

\footnotetext{
*This study is supported by grants from "Europe against Cancer," the Dutch Cancer Society (KWF), and the Prevention Fund of the Netherlands, as well as by an educational grant of Wallac Oy, Finland.

From the Department of Urology and Clinical Chemistry, Erasmus University and Academic Hospital, Rotterdam. The Netherlands

Reprint requests: Chris H. Bangma, M.D., Department of Urology, Academic Hospital, Rotterdam, The Netherlands

Submitted: August 14, 1995, accepted (with revisions): October 16, 1995
}

Several additional parameters, such as prostate volume and age, have been used in an attempt to reduce this unfavorable ratio, without loss of sensitivity. ${ }^{6,7}$

Prostate-specific antigen (PSA) can be found in serum in a free form or may be bound to various serum proteins, predominantly alpha ${ }_{1}$ antichymotrypsin (ACT). ${ }^{8,9}$ The detection of PSA depends on the identification of antigenic epitopes on the PSA molecule by labeled antibodies. Some of these epitopes may be (sterically) obscured by serum proteins, predominantly by $A C T .{ }^{10}$ This introduces the possibility of discriminating between free (F) and complexed PSA, and of calculating the ratio between free and total PSA (F/T ratio). In selected groups the ratio between free and complexed PSA improves the differentiation between prostate carcinoma and benign conditions. ${ }^{11-13}$

The concept of $F / T$ ratio offers a new parameter for the early detection of $P C$ in addition to total serum PSA, digital rectal examination (DRE), and transrectal ultrasonography (TRUS). The question is raised whether the $F / T$ ratio can increase the specificity of existing tests or their combinations in order to reduce the number of negative biopsies, or 
even replace a number of them to make screening less invasive and more cost-effective. To assess the value of the $\mathrm{F} / \mathrm{T}$ ratio, it should be compared to other parameters, such as age, total prostate volume and transition zone volume, total PSA, DRE, and lesions seen by TRUS, which have been used to differentiate between normal and pathologic prostates in screening populations. In men within the PSA range between 4.0 and $10.0 \mathrm{ng} / \mathrm{mL}$ with glands smaller than $50 \mathrm{~mL}$, the $\mathrm{F} / \mathrm{T}$ ratio has been reported to increase the specificity to detect PC. ${ }^{14}$ The various parameters should be analyzed in a representative sample of the male population, independent of the PSA level. The best estimate of the incidence of PC in the general population would be obtained by submitting all participants to sextant prostate biopsies. However, this is not feasible. Assessment of screening modalities, therefore, will always be biased by the method of sampling and by the method of PC detection in the population studied.

\section{MATERIAL AND METHODS}

\section{MATERIAL}

Serum samples, which had been stored at $-70^{\circ} \mathrm{C}$, were used to determine total and free PSA by two DELFIA second generation PSA assays.

The DELFIA ProStatus PSA Free/Total assay provides simultaneous dual label measurement of free and total PSA by using time-resolved fluorometry of europium (free PSA) and samarium chelates (total PSA). The ProStatus PSA EQM provides a highly sensitive single label (europium) assay of total PSA. Both assays measure total PSA with the same reagent combination to give equimolar detection of PSA in free and complexed form. The detection limits for the dual label assay are less than $0.01 \mathrm{ng} / \mathrm{mL}$, and less than $0.1 \mathrm{ng} / \mathrm{mL}$ for free and total PSA, respectively, and less than $0.01 \mathrm{ng} / \mathrm{mL}$ for the single label total PSA assay. Regarding the imprecision of both ProStatus assays, we found coefficients of variation between $7.2 \%$ (at a serum concentration of $0.7 \mathrm{ng} / \mathrm{mL}$ ) and $4.3 \%$ (at a serum concentration of $70.7 \mathrm{ng} / \mathrm{mL}$ ) for total PSA, and between $10.3 \%$ (at a serum concentration of $0.17 \mathrm{ng} / \mathrm{mL}$ ) for free PSA and $3.7 \%$ (at a serum concentration of $35.5 \mathrm{ng} / \mathrm{mL}$ ) during 22 days, with six control samples supplied by the manufacturer.

The correlation coefficient $(r)$ between the ProStatus assays and both the Abbott IMx and the Hybritech Tandem E assays has shown to be better than 0.97 for men with and men without $\mathrm{PC} .{ }^{15,16}$

\section{METHODS}

Two sample collections of men originating from the Rotterdam community were combined for this study: 903 participants of the ERSPC, and 823 men of the Rotterdam feasibility study for screening prostate carcinoma. ${ }^{17}$ All men aged 55 to 77 years had been randomized for total serum PSA determination (Hybritech Tandem E for the ERSPC participants, Abbott IMx for the feasibility study), DRE, and TRUS. Blood sampling was done prior to further testing, and DRE was performed before TRUS by one of five well-trained urology residents without knowledge of the PSA values. An 1846 Bruel and Kjaer $7.0 \mathrm{MHz}$ biplanar ultrasound probe was used for diagnostic ultrasonography, and planimetric volumetry of the prostate gland and its inner zone..$^{18}$ Ultrasound-guided sextant prostatic biopsies were taken in case of an elevated PSA more than $4.0 \mathrm{ng} / \mathrm{mL}$ and/or a suspect DRE, and, in case of hypoechoic lesions seen by TRUS, biopsies of these lesions were taken in addition to sextant biopsies. All men with a negative biopsy, and those in whom no biopsy was taken, were considered free of PC.

The biopsy results were used to assess the capability of the F/T ratio in combination with the total PSA to detect prostate carcinoma, as biopsy illustrated the definite histologic status of the individual.

\section{STATISTICS}

Continuous parameters were compared by means of the Mann-Whitney U test (a two-sided 0.05 level of significance was used). Linear univariate regression analysis of continuous parameters, such as total PSA, free PSA, total prostate volume, volume of the inner zone, and age, was used to express the correlation between factors.

Predictors for a positive biopsy result were estimated as a function of these parameters, and of binary parameters, such as DRE and TRUS, by means of logistic regression analysis. Logistic regression analysis was chosen as the distribution of the values of various parameters was not Gaussian. It was realized that several parameters may show significant correlation to each other. For all men who had biopsies, these parameters were used as input in a backward deletion logistic regression analysis. The output of this procedure is a predictor that specifies the chance on a positive biopsy result as a function of the variables most relevant to this end. All variables with insignificant Wald statistics are deleted. ${ }^{19}$ The relative intrinsic discriminatory potential of these predictors was assessed by comparing receiver operating characteristic (ROC) curves, in which each point of the curve fits a combination of a level of sensitivity with its specificity. ${ }^{20}$ For the predictor with the best properties (this is the largest area under the ROC curve), the predictive value was determined.

In the subgroup of men in the PSA range between 4.0 and $10.0 \mathrm{ng} / \mathrm{mL}$, sensitivity and specificity of the $\mathrm{F} / \mathrm{T}$ ratio to predict prostate carcinoma were calculated for all prostate gland volumes and for those smaller than $50 \mathrm{~mL}$.

\section{RESULTS}

General characteristics of all men are shown in Table I. In 1726 men 67 cancers were histologically diagnosed by 308 sextant prostate biopsies. Planimetric volumetry was available in 1565 benign and 64 malignant prostates. Table II shows the ratio between the biopsies taken and the cancers detected (the $\mathrm{Bx} / \mathrm{PC}$ ratio) for each of the screening modalities, or the four possible combinations. The $\mathrm{Bx} / \mathrm{PC}$ illustrates the number of biopsies needed to detect one cancer and is the reciprocal of the positive predictive value of that screening modality. A suspicious TRUS alone showed the largest number of biopsies needed to detect one carcinoma, while the combination of a suspicious TRUS, an abnormal DRE, and a PSA value of $4.0 \mathrm{ng} / \mathrm{mL}$ or more was the most efficient in detecting $\mathrm{PC}$.

The values of $F / T$ ratio measured by the ProStatus Free/Total PSA assay in relation to men with and without cancer is illustrated in Figure 1 by Box Whisker plots. The box includes the results of $50 \%$ of the participants, with the median value plotted inside. The notch represents the $95 \%$ confidence interval of the median, and the middle 
TABLE I. Age, prostatic volumes, and PSA values of 1659 apparently benign prostates and 67 with prostate cancer

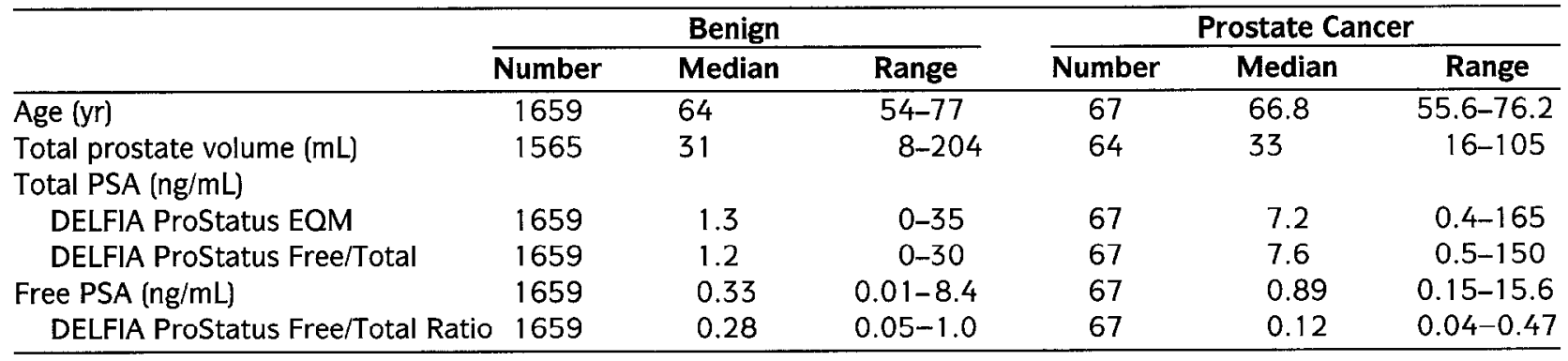

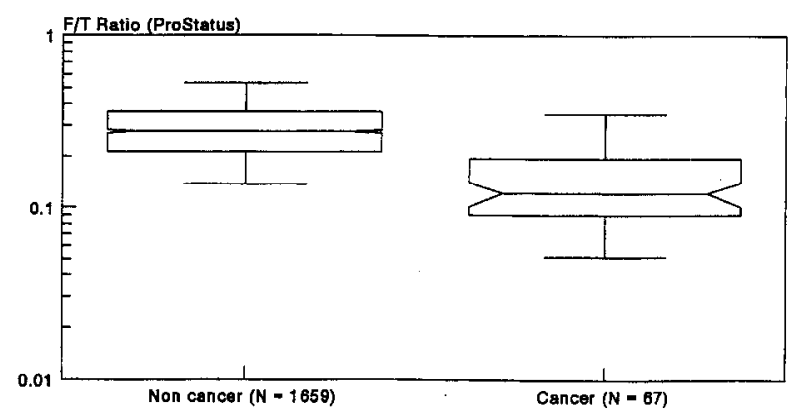

FIGURE 1. The PSA free to total (F/T) ratio in 1659 benign and 67 prostate cancer patients, Box Whisker plots. $\mathrm{P}<0.05$.

vertical line the $90 \%$ interval of all values. The median $\mathrm{F} / \mathrm{T}$ ratio for 1726 benign participants was 0.28 , which significantly differed from the $F / T$ ratio of 0.12 of 67 men with a carcinoma.

In Figure 2 the relationship between $\mathrm{F} / \mathrm{T}$ ratio and the total PSA for 308 men who underwent a prostate biopsy is depicted. It can be seen that the distribution of detected cancers overlaps that of nonmalignancies, especially in the PSA range between 4.0 and $10.0 \mathrm{ng} / \mathrm{mL}$.
TABLE II. Ratio between the biopsies taken and the carcinomas detected (the BX/PC) for each of the screening modalities, or the four possible combinations

\begin{tabular}{lccr}
\hline & No. biopsies & No. PC & Bx/PC \\
\hline TRUS alone & 53 & 2 & 26.5 \\
DRE alone & 29 & 3 & 9.7 \\
PSA alone & 126 & 22 & 5.7 \\
TRUS + DRE & 48 & 7 & 6.9 \\
TRUS + PSA & 11 & 4 & 2.8 \\
DRE + PSA & 11 & 6 & 1.8 \\
TRUS + DRE + PSA & 30 & 23 & 1.3 \\
Total & 308 & 67 & 4.6
\end{tabular}

KEY: $\mathrm{Bx}=$ biopsy $\mathrm{DRE}=$ digital rectal examination $\mathrm{PC}=$ prostate cancer $; \mathrm{TRUS}=$ transrectal ultrasonography.

The relative effectiveness of the diagnostic tests was illustrated in the ROC curves of Figure 3 . These ROC curves show that, with a standard error of the areas of 0.03 , the addition of DRE improved the detection of prostate carcinoma by total serum PSA significantly. Addition of free PSA to the combination of PSA and DRE improved detectability by 1

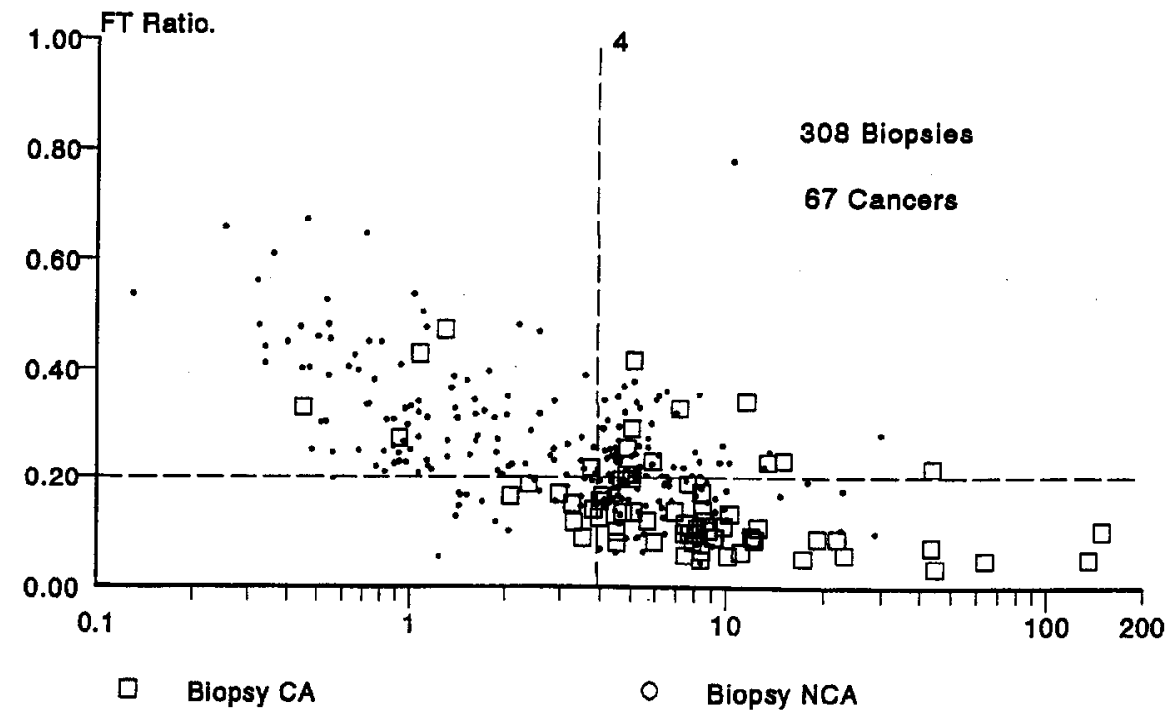

Total PSA, ProStatus PSAF/T [ng/mL]
FIGURE 2. The relationship between free to total $(F / T)$ ratio and the total PSA for 308 men who underwent a prostate biopsy; 67 cancers are indicated. $C A=$ carcinoma, NCA = no carcinoma. 


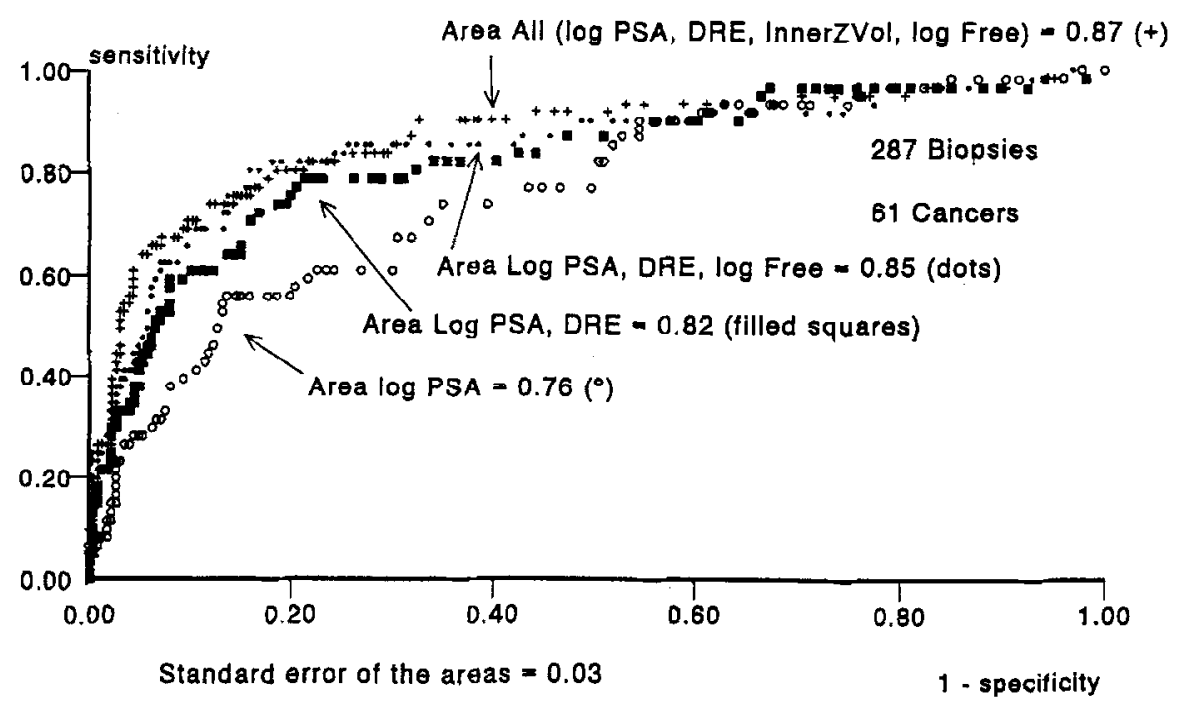

FIGURE 3. The relative effectiveness of diagnostics illustrated in receiver operating characteristic curves of 287 men who underwent prostate biopsies. Standard error of the areas $=0.03 . D R E=$ digital rectal examination; $Z=$ zone.

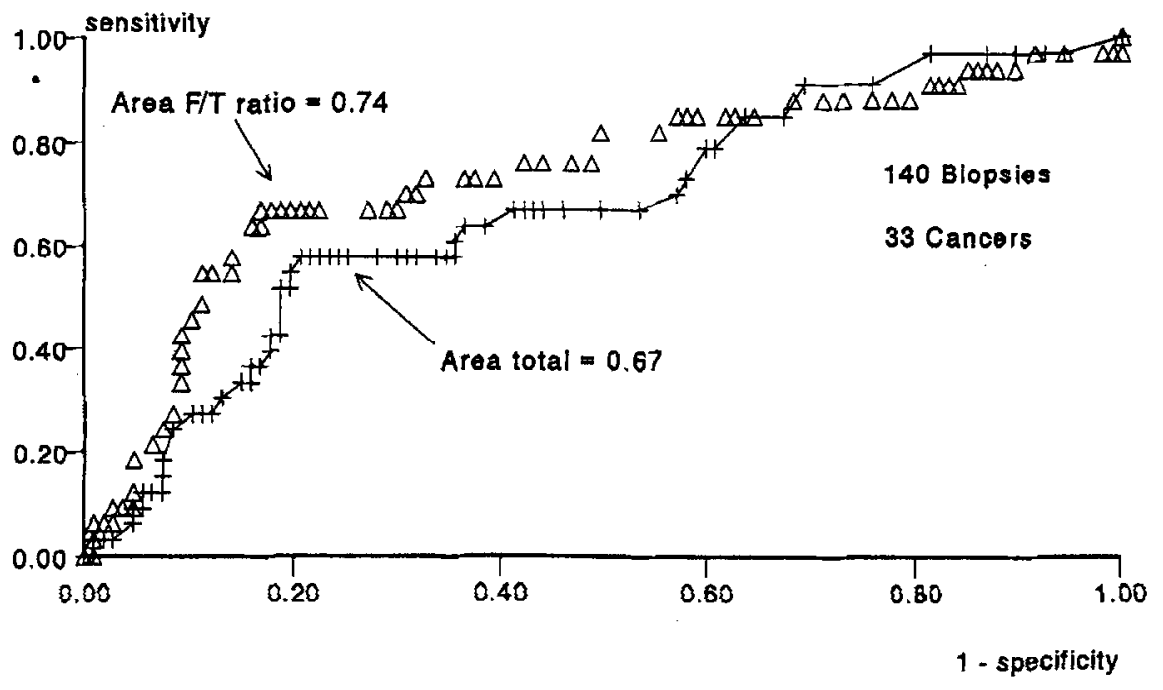

FIGURE 4. The relative effectiveness of diagnostics illustrated in receiver operating characteristic curves of 140 men who underwent prostate biopsies in the total serum PSA range between 4.0 and $10.0 \mathrm{ng} / \mathrm{mL}$. Standard error of the areas $=0.05$. $F / T=$ free to total.

TABLE III. Results of univariate regression analysis: $P<0.01$

\begin{tabular}{|c|c|c|c|c|c|}
\hline \multirow[b]{2}{*}{ Correlation } & \multirow[b]{2}{*}{ Log PSA } & \multirow[b]{2}{*}{ Log Free PSA } & \multicolumn{2}{|c|}{ Volume } & \multirow[b]{2}{*}{ Age } \\
\hline & & & Total Gland & Inner Zone & \\
\hline \multicolumn{6}{|l|}{$\overline{B e n i g n}(n=1542)$} \\
\hline Log PSA & & 0.87 & 0.54 & 0.41 & 0.16 \\
\hline Log free PSA & & & 0.62 & 0.45 & 0.22 \\
\hline Voume total gland & & & & 0.74 & 0.23 \\
\hline Volume inner zone & & & & & 0.22 \\
\hline \multicolumn{6}{|c|}{ Prostate cancer $(n=61)$} \\
\hline Log PSA & & 0.81 & 0.11 & 0.08 & 0.12 \\
\hline Log free PSA & & & 0.46 & 0.32 & 0.31 \\
\hline Volume total gland & & & & 0.70 & 0.26 \\
\hline Volume inner zone & & & & & 0.24 \\
\hline
\end{tabular}

standard error. The best predictor of a positive biopsy was obtained by the combination of total PSA, DRE, inner zone volume, and free PSA.

For the range of total PSA between 4.0 and 10.0 $\mathrm{ng} / \mathrm{mL}$, including 140 prostate hiopsies and 33 PCs, the ROC curves of total serum PSA and of the $F / T$ ratio were constructed to illustrate their relative potential to predict a positive biopsy (Fig. 4 ). The standard error of the areas under the curve was 0.05 . No significant difference between the curves was obtained.

In Table III, the results of the linear univariate regression analysis are shown for 61 men with carcinoma, and 1542 without carcinoma. All correlations were significant. A high correlation existed between total and free PSA. Volumetric parameters correlated slightly better with free PSA compared to total serum PSA. For men with 


\section{TABLE IV. Results of logistic regression} analysis of independent parameters for predicting a positive biopsy in 308 men, first step in backward deletion procedure

\begin{tabular}{lrrrc}
\hline Variable & \multicolumn{1}{c}{ B } & SE & Wald & Significance \\
\hline Log PSA & 5.84 & 0.98 & 35.12 & 0.00 \\
Log free PSA & -2.32 & 1.15 & 4.03 & 0.04 \\
DRE & -0.93 & 0.24 & 14.97 & 0.00 \\
Volume total & -0.01 & 0.01 & 0.58 & 0.44 \\
Volume inner & -0.03 & 0.02 & 3.71 & 0.05 \\
Age & 0.04 & 0.04 & 1.06 & 0.30 \\
TRUS & -0.18 & 0.22 & 0.63 & 0.42
\end{tabular}

KEY: $B=$ predictor for a positive biopsy; $S E=$ standard error of the predictor; Wald statistic = square of the ratio of the predictor to its standard error; significance $=$ Wald statistic, threshold 0.05 .

prostate cancer, the correlation between volumetric parameters and PSA was less compared to men without a carcinoma. Age was uniformly mildly correlated to all other parameters.

In Table IV, the results of logistic regression analysis of the parameters for predicting a positive biopsy in 308 men are shown. Total serum PSA was the best single predictor for prostate carcinoma, followed by DRE. Free PSA and the ultrasonic volume of the inner zone of the prostate were just significant as predictors. Age was the first parameter deleted in the backward stepwise elimination procedure, followed by total prostate volume and TRUS.

Table V shows the calculation of the sensitivity and specificity of the F/T ratio for men in the PSA range between 4.0 and $10.0 \mathrm{ng} / \mathrm{mL}$. In Table V(A), all 140 men are included; in Table V(B), the 81 men with a planimetric prostate gland volume of less than $50 \mathrm{~mL}$ are included. The standard errors were calculated to illustrate the confidence ranges $( \pm 2 \mathrm{SE})$ of the chosen sensitivity and specificity levels. For an $\mathrm{F} / \mathrm{T}$ ratio cutoff value of 0.20 , a sensitivity level of $82 \%$ (95\% confidence range of $64 \%$ to $100 \%$ ) and a specificity of $51 \%$ (range, $43 \%$ 10 59\%) were found. In the volume-restricted group, the sensitivity at the same cutoff value was approximately $90 \%$ (Table VB), and the specificity was $49 \%$ (range, $37 \%$ to $61 \%$ ). The small numbers ( 22 cancers in 81 men, or $5 \%$ of the studied population) introduced wide confidence ranges.

\section{COMMENT}

For the detection of PC currently, the combination of serum PSA, DRE, and TRUS is available. Detection rates in community-based populations vary between $2 \%$ and $5 \%$. In this study the detection rate was $3.3 \%$. A combination of total serum PSA, DRE, and TRUS as indicators for biopsy led to a subset of men in whom 1.3 sextant prostate biopsies were needed to detect one carcinoma. The mean $\mathrm{Bx} / \mathrm{PC}$ ratio was 4.6 . These ratios correspond very well to those found by Catalona et $a .^{5}$ in a screening study of 6630 men evaluated by PSA and DRE. In this study it was also found that the $\mathrm{Bx} / \mathrm{PC}$ ratio is relatively constant across age. Further clarifying information on the optimal use of the available screening tests will, it is hoped, be an early result of the ERSPC.

Serum PSA is the best single screening modality for prostate carcinoma available, as shown in Table II. Logistic regression analysis could illustrate the value of various parameters, which were no indicators for sextant biopsy in this study (Table IV). Due to the high correlation between total serum and free PSA, much of the influence of free PSA as a predictor was reduced by total serum PSA. Due to the higher correlation between total or free PSA and the total gland volume, this volumetric parameter was replaced by the inner zone volume in the backward elimination procedure. A combination of the modalities of serum PSA and DRE, with others, such as free PSA or the volume of the inner zone of the prostate, may add to the specificity and sensitivity of the detection, as illustrated by an increasing area under the ROC curve (Fig. 3). DRE increased the area of total serum PSA by 2 SE, whereas the combination of DRE and free PSA increased the area by $3 \mathrm{SE}$. It is suggestive, although not statistically significant, that the application of the F/T ratio in addition to total serum PSA in combination with DRE

TABLE V. Sensitivity and specificity of the free to total ratio to detect prostate cancer

\begin{tabular}{|c|c|c|c|c|}
\hline Sensitivity & SE & Specificity & SE & Free to Total Ratio Cutoff \\
\hline \multicolumn{5}{|c|}{ A: PSA 4 to $10 \mathrm{ng} / \mathrm{mL}(\mathrm{n}=140, \mathrm{PC}=33)$} \\
\hline 100 & & 0 & & 0.41 \\
\hline 91 & 2 & 19 & 2 & 0.28 \\
\hline 82 & 9 & 51 & 4 & 0.20 \\
\hline 71 & 8 & 68 & 4 & 0.17 \\
\hline \multicolumn{5}{|c|}{ B: PSA 4 to $10 \mathrm{ng} / \mathrm{mL}$, gland volume $<50 \mathrm{~mL}(\mathrm{n}=81, \mathrm{PC}=22$ ) } \\
\hline 100 & & 39 & 6 & 0.21 \\
\hline 91 & 6 & 49 & 6 & 0.19 \\
\hline 82 & 9 & 74 & 6 & 0.14 \\
\hline 73 & 10 & 82 & 5 & 0.12 \\
\hline
\end{tabular}


for screening for PC is useful. No optimal analysis could be performed in the total PSA range below $4.0 \mathrm{ng} / \mathrm{mL}$, as biopsies were taken only on the indication of TRUS or DRE. For the total PSA range between 4.0 and $10.0 \mathrm{ng} / \mathrm{mL}$, however, no significant difference between the ROC curves was obtained in this study.

The use of TRUS for prostate volumetry and lesion detection in screening for prostate cancer does not appear to be cost-effective. Ultrasonic volume of the inner zone (as a representation of the prostatic hyperplasia), only marginally adds to the prediction of a positive biopsy. Calculation of sensitivity and specificity in glands restricted to a volume of $50 \mathrm{~mL}$ or less in the gray PSA range between 4.0 and $10.0 \mathrm{ng} / \mathrm{mL}$ did not reveal statistically significant improvement in specificity compared to the whole group in this PSA range. Lesion detection by TRUS did not have significant predictive value in the logistic regression analysis. This means that the two carcinomas detected by TRUS (Table II) could have been detected by use of the free PSA or the inner zone volume. The logistic regression does not illustrate the number of biopsies needed for the detection of those two carcinomas, but from Table II it can be seen that 26.5 biopsies per cancer found were needed to determine histologically the cause of those hypoechogenic lesions.

\section{CONCLUSIONS}

The combination of total serum PSA and DRE remains the standard for detection of prostate carcinoma in a screening population. Their specificity may be improved minimally by the F/T ratio, and statistical significance might be reached in a population larger than 1726 men. The threshold of the F/T ratio, and the PSA range for its application, remains to be assessed prospectively.

ACKNOWLEDGMENT. To our laboratory technicians Ineke Eman and Bianca E. den Hartog for their expert work.

\section{REFERENCES}

1. Mettlin C, Lee F, Drago J, and Murphy GP: The American Cancer Society National Prostate Cancer Detection Project, findings on the detection of early prostate cancer in 2425 men. Cancer 67: 2949-2958, 1991 .

2. Brawer MK, Chetner MP, Beatie J, Buchner DM, Vessella RL, and Lange PH: Screening for prostatic carcinoma with prostate specific antigen. J Urol 147: 841-845, 1992.

3. Labrie F, Dupont A, Suburu R, Cusan L, Tremblay M, Gomez JL, and Emond J: Serum prostate specific antigen as pre-screening test for prostate cancer. J Urol 147: 846-852, 1992.

4. Kirkels WJ, Schröder FH, Damhuis RS, Nijs HG, and Roobol-Bouts M: A European study for screening of adenocarcinoma of the prostate. Report of a pilot study. J Urol 149: 289A, 1993.

5. Catalona WJ, Richie JP, Ahmann FR, Hudson MA, Scardino PT, Flanigan RC, deKernion JB, Ratliff TL, Kavoussi
LR, Dalkin BL, et al: Comparison of digital rectal examination and serum prostate specific antigen in early detection of prostate cancer: results of multicenter clinical trial of 6630 men. J Urol 151: 1283-1290, 1994.

6. Littrup PJ, Kane RA, Mettlin CJ, Murphy GP, Lee F, Toi A, Badalament $R$, and Babaian $R$ : Cost-effective prostate cancer detection. Reduction of low-yield biopsies. The investigators of the American Cancer Society National Prostate Cancer Detection Project. Cancer 74: 3146-3158, 1994.

7. Oesterling JE, Jacobsen SJ, and Cooner WH: The use of age-specific reference ranges for serum prostate specific antigen in men 60 years old or older. J Urol 153: 1160-1163, 1995.

8. Stenman UH, Leinonen J, Alfthan H, Rannikko S, Tuhkanen K, and Alfthan O: A complex between prostatespecific antigen and alpha 1-antichymotrypsin is the major form of prostate-specific antigen in serum of patients with prostatic cancer: assay of the complex improves clinical sensitivity for cancer. Cancer Res 51: 222-226, 1991.

9. Lilja $\mathrm{H}$, Christensson A, Dahlen U, Matikainen MT, Nilsson O, Petterson K, and Lovgren T: Prostate-specific antigen in serum occurs predominantly in complex with alpha 1-antichymotrypsin. Clin Chem 37: 1618-1625, 1991.

10. Lilja H, Cockett AT, and Abrahamsson PA: Prostate specific antigen predominantly forms a complex with alpha 1-antichymotrypsin in blood. Implications for procedures to measure prostate specific antigen in serum. Cancer 70: 230-234, 1992.

11. Christensson A, Bjork T, Nilsson O, Dahlen U, Matikainen MT, Cockett AT, Abrahamsson PA, and Lilja $\mathrm{H}$ : Serum prostate-specific antigen complexed to alpha-1antichymotrypsin as an indicator of prostate cancer. J Urol 150: 100-105, 1993.

12. Lilja H, Bjork T, Abrahamsson PA, Stenman U, Shaw N, Dowell B, Oesterling J, Pettersson K, Piironen T, and Lovgren $T$ : Improved separation between normals, benign prostatic hyperplasia (BPH), and carcinoma of the prostate (CAP) by measuring free $(\mathrm{F})$, complexed $(\mathrm{C})$ and total concentrations (T) of prostate specific antigen (PSA) (AUA abstract 692). J Urol 151: 400A, 1994.

13. King C, Friese J, Lauren L, Dowell B, Shaw N, Lilja H, Stenman U, Piironen T, and Pettersson K: Measurement on IMx of free and total forms of prostate specific antigen for differentiation of patients with benign prostatic hyperplasia and prostate cancer. Clin Chem 40: 1007-1010, 1994.

14. Catalona WJ, Smith DS, Wolfert RL, Wang TS, Rittenhouse HG, and Ratliff TL: Increased specificity of PSA screening through measurement of percent free PSA in serum. J Urol 153: 336A, 1995.

15. Bangma $\mathrm{CH}$, Kranse R, Blijenberg BG, and Schröder FH: The new DELFIA prostate specific antigen (PSA): results of the first comparative evaluation. J Urol 153 (suppl): 294A, 1995.

16. Blijenberg BG, Bangma $\mathrm{CH}$, Kranse $\mathrm{R}$, and Schröder FH: Analytical evaluation of the new DELFIA free and total prostate-specific antigen assays. 11 th International European Congress of Clinical Chemistry, Tampere, 1995.

17. Schröder FH, Denis LJ, Kirkels W, De Koning HJ, and Standaert B: European Randomized Study of Screening for Prostate Cancer, progress report of Antwerp and Rotterdam pilot studies. Cancer 76(1) 129-134, 1995.

18. Niemer AQHJ, Davidson PJT, Bangma $\mathrm{CH}$, Hop WCJ, and Schröder FH: Observations on the reliability and reproducibility of transrectal ultrasonic volume measurements of the prostate: the effect of equipment and observers. Br J Urol 1995 (in press).

19. Norusis MJ: SPSS/PC+ Advanced Statistics 4.0. SPSS Inc., Chicago, b1-b37.

20. Green D, and Swets J: Signal detection theory and psychognosics. New York, John Wiley \& Sons, 1996, pp 45-49. 